\title{
NONLINEAR DELAY REACTION-DIFFUSION SYSTEMS WITH NONLOCAL INITIAL CONDITIONS HAVING AFFINE GROWTH
}

\author{
Monica-Dana Burlic $\breve{A}$ - Daniela Roşu
}

(Submitted by W. Kryszewski)

ABstract. We consider a class of abstract evolution reaction-diffusion systems with delay and nonlocal initial data of the form

$$
\begin{cases}u^{\prime}(t) \in A u(t)+F\left(t, u_{t}, v_{t}\right) & \text { for } t \in \mathbb{R}_{+}, \\ v^{\prime}(t) \in B v(t)+G\left(t, u_{t}, v_{t}\right) & \text { for } t \in \mathbb{R}_{+}, \\ u(t)=p(u, v)(t) & \text { for } t \in\left[-\tau_{1}, 0\right], \\ v(t)=q(u, v)(t) & \text { for } t \in\left[-\tau_{2}, 0\right],\end{cases}
$$

where $\tau_{i} \geq 0, i=1,2, A$ and $B$ are two $m$-dissipative operators acting in two Banach spaces, the perturbations $F$ and $G$ are continuous, while the history functions $p$ and $q$ are nonexpansive functions with affine growth. We prove an existence result of $C^{0}$-solutions for the above problem and we give an example to illustrate the effectiveness of our abstract theory.

\section{Introduction}

Let $X, Y$ be Banach spaces, $\tau_{1}, \tau_{2} \geq 0$, and let $A: D(A) \subseteq X \rightsquigarrow X$ and $B: D(B) \subseteq Y \rightsquigarrow Y$ be $m$-dissipative operators. Our paper is devoted to provide

2010 Mathematics Subject Classification. Primary: 34G25, 34K30; Secondary: 35K55, $35 \mathrm{~K} 57,47 \mathrm{H} 06$.

Key words and phrases. Differential delay evolution systems; nonlocal delay initial condition; metric fixed point arguments; nonlinear reaction-diffusion systems.

This work was supported by a grant of the Romanian National Authority for Scientific Research, CNCS-UEFISCDI, project number PN-II-ID-PCE-2011-3-0052. 
an existence result for $C^{0}$-solutions to the next reaction-diffusion system with delay and nonlocal initial conditions:

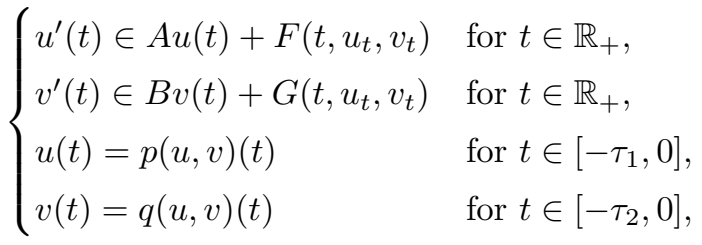

where the perturbations $F: \mathbb{R}_{+} \times C\left(\left[-\tau_{1}, 0\right] ; \overline{D(A)}\right) \times C\left(\left[-\tau_{2}, 0\right] ; \overline{D(B)}\right) \rightarrow X$ and $G: \mathbb{R}_{+} \times C\left(\left[-\tau_{1}, 0\right] ; \overline{D(A)}\right) \times C\left(\left[-\tau_{2}, 0\right] ; \overline{D(B)}\right) \rightarrow Y$ are continuous and the initial data $p: C_{\mathrm{b}}\left(\left[-\tau_{1},+\infty\right) ; \overline{D(A)}\right) \times C_{\mathrm{b}}\left(\left[-\tau_{2},+\infty\right) ; \overline{D(B)}\right) \rightarrow C\left(\left[-\tau_{1}, 0\right] ; X\right)$ and $q: C_{\mathrm{b}}\left(\left[-\tau_{1},+\infty\right) ; \overline{D(A)}\right) \times C_{\mathrm{b}}\left(\left[-\tau_{2},+\infty\right) ; \overline{D(B)}\right) \rightarrow C\left(\left[-\tau_{2}, 0\right] ; Y\right)$ are nonexpansive functions with affine growth.

Partial differential equations with nonlocal initial conditions arise in many areas of applied mathematics and represent mathematical models of various phenomena. See Deng [18] and McKibben [25]. The study for nonlocal Cauchy problems without delay was initiated by Byszewski [15] (in the semilinear case), and subsequently it has been developed by many authors. We mention here some significant contributions to the field: Aizicovici and Lee [1], Aizicovici and McKibben [2], García-Falset [21], García-Falset and Reich [22], Cardinali, Precup and Rubbioni [16] in the single-valued case, Aizicovici and Staicu [3], Paicu and Vrabie [32], Zhu and $\mathrm{Li}$ [43] in the multi-valued case. Nica [31] proved the existence of the solutions for nonlinear first order differential systems with nonlocal conditions. These results were extended by Bolojan-Nica, Infante and Precup [7] to differential systems with nonlinear and nonlocal boundary conditions. For delay evolution equations with local initial conditions see Mitidieri and Vrabie [26], [27], Necula and Popescu [28], and the references therein. As far as nonlocal initial conditions are concerned, we mention the papers Burlică and Roşu [11], Burlică, Roşu and Vrabie [13], Necula, Popescu and Vrabie [29], Vrabie [37]-[41], Wang and Zhu [42]. For parabolic systems with nonlinear, nonlocal initial conditions we mention the paper of Infante and Maciejewski [24]. Concerning the reaction-diffusion systems without delay see: Burlică [8], Burlică and Roşu [9], [10], Díaz and Vrabie [19], Necula and Vrabie [30], Roşu [33], [34]. Existence results for reaction-diffusion systems with delay and nonlocal initial conditions were obtained in Burlică, Roşu and Vrabie [14] for the single-valued case and by Burlică and Roşu [12] for the multi-valued case. The present work complements Burlică, Roşu and Vrabie [14] by allowing the nonlocal initial constraint function $p$ to have affine instead of linear growth with respect to the first argument and $q$ to obey the same property with respect to its second variable. Moreover, we allow the unknown functions to have different delays, $\tau_{1}$ and $\tau_{2}$. Our general assumptions include reaction-diffusion systems in which one or both 
perturbations are of the form $F\left(t, u(t), v_{t}\right)$ or $G\left(t, u_{t}, v(t)\right)$. So, the initial nonlocal constraint $p$ belongs to a general class of functions including the important instances below:

(i) $p(u, v)(t)=p_{0}(u)(t)+\varphi(t), t \in\left[-\tau_{1}, 0\right]$, where $\varphi \in C\left(\left[-\tau_{1}, 0\right] ; X\right)$ and $p_{0}: C_{\mathrm{b}}\left(\left[-\tau_{1},+\infty\right) ; \overline{D(A)}\right) \rightarrow C\left(\left[-\tau_{1}, 0\right] ; X\right) ;$

(ii) $p(u, v)(t)=u(t+2 \pi)$ or $p(u, v)(t)=-u(t+2 \pi), t \in\left[-\tau_{1}, 0\right]$;

(iii) $p(u, v)(t)=\int_{\tau_{1}}^{+\infty} k_{1}(s) u(s+t) d s, t \in\left[-\tau_{1}, 0\right]$, where $k_{1} \in L^{1}\left(\tau_{1},+\infty ; \mathbb{R}\right)$ with $\int_{\tau_{1}}^{+\infty}\left|k_{1}(s)\right| d s=1$.

Similar remarks refer to the nonlocal initial constraint function $q$ and, of course, we can consider various mixed conditions on the pair $(p, q)$.

Even though our main result is inspired by Vrabie [41], we emphasize that it cannot be obtained by a direct application of the above mentioned result in a product space simply because our assumptions lead to a problem essentially different from that in Vrabie [41]. Finally, the passing from linear to affine growth is not at all so simple as it seems to be at first glance because in the latter case we face some real difficulties in obtaining the sharp estimates needed in the proof.

The paper is organized as follows. Section 2 contains the basic background material on $m$-dissipative operators and evolution equations. The main existence result for problem (1.1) is stated in Section 3. Section 4 is devoted to some auxiliary results while the proof of the main result of this paper is carried out in Section 5. Finally, Section 6 contains an example to which our abstract theory does apply but the previous known results do not.

\section{Preliminaries}

As usual, $\mathbb{R}_{+}$denotes the set of all nonnegative real numbers. Let $X$ be a real Banach space with the norm $\|\cdot\|$ and let $I$ be a real interval. We denote by $C_{\mathrm{b}}(I ; X)$ the space of all bounded and continuous functions from $I$ to $X$, equipped with the sup-norm $\|\cdot\|_{C_{\mathrm{b}}(I ; X)}=\sup \{\|u(t)\| ; t \in I\}$. If the interval $I$ is compact we use the standard notation $C(I ; X)$ for the space and $\|\cdot\|_{C(I ; X)}$ for the sup-norm. If $Z \subset X$ is a closed subset, we denote by $C_{\mathrm{b}}(I ; Z)$ the closed subset in $C_{\mathrm{b}}(I ; X)$ consisting of all elements $u \in C_{\mathrm{b}}(I ; X)$ satisfying $u(t) \in Z$ for each $t \in I$.

We denote by $\widetilde{C}_{\mathrm{b}}([a,+\infty) ; X), a \in \mathbb{R}_{+}$, the space $C_{\mathrm{b}}([a,+\infty) ; X)$ endowed with the family of seminorms $\left\{\|\cdot\|_{k} ; k \in \mathbb{N}, k>a\right\},\|u\|_{k}:=\sup \{\|u(t)\|$; $t \in[a, k]\}$, for each $k \in \mathbb{N}, k>a$, and each $u \in C_{\mathrm{b}}([a,+\infty) ; X)$. Thus $\widetilde{C}_{\mathrm{b}}([a,+\infty) ; X)$ is a separated locally convex space and its topology coincides with the uniform convergence on compacta topology.

If $\tau \geq 0, u \in C([-\tau,+\infty) ; X)$ and $t \in \mathbb{R}_{+}$, we denote by $u_{t}:[-\tau, 0] \rightarrow X$ the delayed function defined as $u_{t}(s):=u(t+s)$ for each $s \in[-\tau, 0]$. So, if $\tau=0$, we have $u_{t}(s)=u(t)$ for each $s \in[-\tau, 0]$, simply because $[-\tau, 0]=\{0\}$. 
We assume that the reader is familiar with the theory of $m$-dissipative operators and nonlinear evolution equations in Banach spaces. For the sake of clarity and easy references we recall however some basic concepts and results belonging to this theory. For more details we refer to Barbu [5] and Vrabie [36]. As far as differential equations with delay are concerned, we refer to Hale [23].

Let $X$ be a real Banach space with the norm $\|\cdot\|$. As usual, for $x, y \in X$ we denote by $[x, y]_{+}$the right directional derivative of the norm calculated at $x$ in the direction $y$, i.e.,

$$
[x, y]_{+}:=\lim _{h \downarrow 0} \frac{1}{h}(\|x+h y\|-\|x\|),
$$

and we remark that $\left|[x, y]_{+}\right| \leq\|y\|$. For other significant properties of the mapping $(x, y) \mapsto[x, y]_{+}$, see Barbu [5, Proposition 3.7, p. 103]. A multi-valued operator $A: D(A) \subseteq X \rightsquigarrow X$ is called dissipative if for each $x_{i} \in D(A)$ and $y_{i} \in A x_{i}, i=1,2$, we have $\left[x_{1}-x_{2}, y_{2}-y_{1}\right]_{+} \geq 0$. It is called $m$-dissipative if it is dissipative, and, in addition, $R(I-\lambda A)=X$ for each $\lambda>0$.

Let us consider the evolution equation

$$
u^{\prime}(t) \in A u(t)+f(t), \quad t \in[a, b],
$$

where $f \in L^{1}(a, b ; X)$. A function $u:[a, b] \rightarrow \overline{D(A)}$ is called $a C^{0}$-solution, or integral solution of $(2.1)$ on $[a, b]$, if $u \in C([a, b] ; X)$ and it satisfies

$$
\|u(t)-x\| \leq\|u(s)-x\|+\int_{s}^{t}[u(\theta)-x, f(\theta)+y]_{+} d \theta
$$

for each $x \in D(A), y \in A x$ and $a \leq s \leq t \leq b$.

Theorem 2.1. Let $A: D(A) \subseteq X \rightsquigarrow X$ be $m$-dissipative and $\omega>0$ be such that $A+\omega I$ is dissipative. Then, for each $\xi \in \overline{D(A)}$ and $f \in L^{1}(a, b ; X)$ there exists a unique $C^{0}$-solution of $(2.1)$ on $[a, b]$ which satisfies $u(a)=\xi$. If $f, g \in L^{1}(a, b ; X)$ and $u, v$ are two $C^{0}$-solutions of $(2.1)$ corresponding to $f$ and $g$ respectively, then

$$
\|u(t)-v(t)\| \leq e^{-\omega(t-s)}\|u(s)-v(s)\|+\int_{s}^{t} e^{-\omega(t-\theta)}\|f(\theta)-g(\theta)\| d \theta
$$

for each $a \leq s \leq t \leq b$. In particular, if $x \in D(A)$ and $y \in A x$, we have

$$
\|u(t)-x\| \leq e^{-\omega(t-s)}\|u(s)-x\|+\int_{s}^{t} e^{-\omega(t-\theta)}\|f(\theta)+y\| d \theta
$$

for each $a \leq s \leq t \leq b$.

See Benilan [6] or Barbu [5, Theorem 4.1, p. 128].

For $\xi \in \overline{D(A)}$, we denote by $u(\cdot, a, \xi, f)$ the unique $C^{0}$-solution of $(2.1)$ which satisfies the initial condition $u(a, a, \xi, f)=\xi$. If $\{S(t): \overline{D(A)} \rightarrow \overline{D(A)}, t \geq 0\}$ is the semigroup of nonexpansive mappings generated by $A$ on $\overline{D(A)}$ via the 
Crandall-Liggett Exponential Formula [17], then $S(t) \xi=u(t, 0, \xi, 0)$ for each $\xi \in \overline{D(A)}$ and $t \geq 0$.

The semigroup generated by $A$ is called compact if for each $t>0$ the operator $S(t): \overline{D(A)} \rightarrow \overline{D(A)}$ is compact, i.e., for each bounded set $U \subseteq \overline{D(A)}, S(t)(U)$ is a relatively compact set in $X$.

A subset $\mathcal{F}$ in $L^{1}(a, b ; X)$ is called uniformly integrable if for each $\varepsilon>0$ there exists $\delta(\varepsilon)>0$ such that for each measurable subset $E$ in $[a, b]$ whose Lebesgue measure $\mu(E)<\delta(\varepsilon)$ we have

$$
\int_{E}\|f(s)\| d s \leq \varepsilon
$$

uniformly for $f \in \mathcal{F}$. We remark that if for some $p \in(1,+\infty], \mathcal{F}$ is a bounded subset in $L^{p}(a, b ; X)$, then $\mathcal{F}$ is uniformly integrable.

We will need the compactness result below.

Theorem 2.2. Let $A: D(A) \subseteq X \rightsquigarrow X$ be an m-dissipative operator generating a compact semigroup. Let $\mathcal{D} \subseteq \overline{D(A)}$ be a bounded set and $\mathcal{F}$ in $L^{1}(a, b ; X)$ be an uniformly integrable set. Then, for each $c \in(a, b)$, the $C^{0}$-solutions set

$$
\{u(\cdot, a, \xi, f) ;(\xi, f) \in \mathcal{D} \times \mathcal{F}\}
$$

is relatively compact in $C([c, b] ; X)$. If, in addition, $\mathcal{D}$ is relatively compact in $X$, then the $C^{0}$-solutions set is relatively compact even in $C([a, b] ; X)$.

See Baras [4] or Vrabie [36, Theorem 2.3.3, p. 47].

We also recall the Tychonoff Fixed Point Theorem.

Theorem 2.3. Let $X$ be a separated locally convex topological vector space and $\mathcal{C}$ be a nonempty, convex, and closed subset in $X$. If $\Gamma: \mathcal{C} \rightarrow \mathcal{C}$ is continuous and $\Gamma(\mathcal{C})$ is relatively compact, then it has at least one fixed point, i.e., there exists $\xi \in \mathcal{C}$ such that $\Gamma(\xi)=\xi$.

See Tychonoff [35] or Edwards [20, Theorem 3.6.1, p. 161].

\section{The main result}

In order to state our main result we need the following assumptions.

$\left(\mathrm{H}_{A}\right)$ The operator $A: D(A) \subseteq X \rightsquigarrow X$ is $m$-dissipative, $0 \in D(A), 0 \in A 0$, $\overline{D(A)}$ is convex and there exists $\omega>0$ such that $A+\omega I$ is dissipative.

$\left(\mathrm{H}_{B}\right)$ The operator $B: D(B) \subseteq Y \rightsquigarrow Y$ is $m$-dissipative, $0 \in D(B), 0 \in B 0$, $\overline{D(B)}$ is convex, there exists $\gamma>0$ such that $B+\gamma I$ is dissipative. In addition, the semigroup generated by $B$ on $\overline{D(B)}$ is compact.

$\left(\mathrm{H}_{F}\right)$ The function $F: \mathbb{R}_{+} \times C\left(\left[-\tau_{1}, 0\right] ; \overline{D(A)}\right) \times C\left(\left[-\tau_{2}, 0\right] ; \overline{D(B)}\right) \rightarrow X$ is continuous and satisfies 
$\left(\mathrm{F}_{1}\right)$ there exists $\ell>0$ such that

$\|F(t, u, v)-F(t, \widetilde{u}, \widetilde{v})\| \leq \ell \max \left\{\|u-\widetilde{u}\|_{C\left(\left[-\tau_{1}, 0\right] ; X\right)},\|v-\widetilde{v}\|_{C\left(\left[-\tau_{2}, 0\right] ; Y\right)}\right\}$

for each $(t, u, v),(t, \widetilde{u}, \widetilde{v}) \in \mathbb{R}_{+} \times C\left(\left[-\tau_{1}, 0\right] ; \overline{D(A)}\right) \times C\left(\left[-\tau_{2}, 0\right] ; \overline{D(B)}\right)$;

$\left(\mathrm{F}_{2}\right)$ there exists $m>0$ such that $\|F(t, 0,0)\| \leq m$ for each $t \in \mathbb{R}_{+}$.

$\left(\mathrm{H}_{G}\right)$ The function $G: \mathbb{R}_{+} \times C\left(\left[-\tau_{1}, 0\right] ; \overline{D(A)}\right) \times C\left(\left[-\tau_{2}, 0\right] ; \overline{D(B)}\right) \rightarrow Y$ is continuous and satisfies

$\left(\mathrm{G}_{1}\right)$ with $\ell>0$ and $m>0$ given by $\left(F_{1}\right)$ and $\left(F_{2}\right)$, we have

$\|G(t, u, v)\| \leq \ell \max \left\{\|u\|_{C\left(\left[-\tau_{1}, 0\right] ; X\right)},\|v\|_{C\left(\left[-\tau_{2}, 0\right] ; Y\right)}\right\}+m$

for each $(t, u, v) \in \mathbb{R}_{+} \times C\left(\left[-\tau_{1}, 0\right] ; \overline{D(A)}\right) \times C\left(\left[-\tau_{2}, 0\right] ; \overline{D(B)}\right)$;

$\left(\mathrm{G}_{2}\right)$ for each $T>0$, the family $\{G(t, \cdot, \cdot) ; t \in[0, T]\}$ is uniformly equicontinuous on $C\left(\left[-\tau_{1}, 0\right] ; \overline{D(A)}\right) \times C\left(\left[-\tau_{2}, 0\right] ; \overline{D(B)}\right)$, i.e., for each $\varepsilon>0$ there exists $\eta(\varepsilon)>0$ such that for each $(t, u, v)$ and $(t, \widetilde{u}, \widetilde{v})$ in $[0, T] \times C\left(\left[-\tau_{1}, 0\right] ; \overline{D(A)}\right) \times C\left(\left[-\tau_{2}, 0\right] ; \overline{D(B)}\right)$ satisfying

$$
\|u-\widetilde{u}\|_{C\left(\left[-\tau_{1}, 0\right] ; X\right)} \leq \eta(\varepsilon) \quad \text { and } \quad\|v-\widetilde{v}\|_{C\left(\left[-\tau_{2}, 0\right] ; Y\right)} \leq \eta(\varepsilon)
$$

we have $\|G(t, u, v)-G(t, \widetilde{u}, \widetilde{v})\| \leq \varepsilon$.

$\left(\mathrm{H}_{c}\right)$ The constants $\ell$ and $\delta:=\min \{\omega, \gamma\}$ satisfy the nonresonance condition: $\ell<\delta$.

$\left(\mathrm{H}_{p}\right)$ The function

$p: C_{\mathrm{b}}\left(\left[-\tau_{1},+\infty\right) ; \overline{D(A)}\right) \times C_{\mathrm{b}}\left(\left[-\tau_{2},+\infty\right) ; \overline{D(B)}\right) \rightarrow C\left(\left[-\tau_{1}, 0\right] ; \overline{D(A)}\right)$

satisfies

$\left(\mathrm{p}_{1}\right)$ there exists $a>0$ such that, with $m>0$ given by $\left(\mathrm{F}_{2}\right)$, we have

$$
\|p(u, v)\|_{C\left(\left[-\tau_{1}, 0\right] ; X\right)} \leq\|u\|_{C_{\mathrm{b}}([a,+\infty) ; X)}+m
$$

for each $(u, v) \in C_{\mathrm{b}}\left(\left[-\tau_{1},+\infty\right) ; \overline{D(A)}\right) \times C_{\mathrm{b}}\left(\left[-\tau_{2},+\infty\right) ; \overline{D(B)}\right)$;

$\left(\mathrm{p}_{2}\right)$ there exists $d>a$, where $a$ is given by $\left(\mathrm{p}_{1}\right)$, such that we have

$\|p(u, v)-p(\widetilde{u}, \widetilde{v})\|_{C\left(\left[-\tau_{1}, 0\right] ; X\right)} \leq \max \left\{\|u-\widetilde{u}\|_{C([a, d] ; X)},\|v-\widetilde{v}\|_{C([a, d] ; Y)}\right\}$

for each $(u, v),(\widetilde{u}, \widetilde{v}) \in C_{\mathrm{b}}\left(\left[-\tau_{1},+\infty\right) ; \overline{D(A)}\right) \times C_{\mathrm{b}}\left(\left[-\tau_{2},+\infty\right) ; \overline{D(B)}\right)$.

$\left(\mathrm{H}_{q}\right)$ With $a$ given by $\left(\mathrm{p}_{1}\right)$ and $m$ given by $\left(\mathrm{F}_{2}\right)$, the function

$q: C_{\mathrm{b}}\left(\left[-\tau_{1},+\infty\right) ; \overline{D(A)}\right) \times C_{\mathrm{b}}\left(\left[-\tau_{2},+\infty\right) ; \overline{D(B)}\right) \rightarrow C\left(\left[-\tau_{2}, 0\right] ; \overline{D(B)}\right)$

satisfies

(q1) for each $(u, v) \in C_{\mathrm{b}}\left(\left[-\tau_{1},+\infty\right) ; \overline{D(A)}\right) \times C_{\mathrm{b}}\left(\left[-\tau_{2},+\infty\right) ; \overline{D(B)}\right)$, we have

$$
\|q(u, v)\|_{C\left(\left[-\tau_{2}, 0\right] ; Y\right)} \leq\|v\|_{C_{\mathrm{b}}\left(\mathbb{R}_{+} ; Y\right)}+m ;
$$


$\left(\mathrm{q}_{2}\right)$ for each $(u, v),(\widetilde{u}, \widetilde{v}) \in C_{\mathrm{b}}\left(\left[-\tau_{1},+\infty\right) ; \overline{D(A)}\right) \times C_{\mathrm{b}}\left(\left[-\tau_{2},+\infty\right) ; \overline{D(B)}\right)$, we have

$$
\begin{aligned}
\| q(u, v)-q(\widetilde{u}, \widetilde{v}) & \|_{C\left(\left[-\tau_{2}, 0\right] ; Y\right)} \\
& \leq \max \left\{\|u-\widetilde{u}\|_{C_{\mathrm{b}}([a,+\infty) ; X)},\|v-\widetilde{v}\|_{C_{\mathrm{b}}([a,+\infty) ; Y)}\right\} ;
\end{aligned}
$$

( $\left.\mathrm{q}_{3}\right)$ for each bounded set $\mathcal{U}$ in $C_{\mathrm{b}}\left(\mathbb{R}_{+} ; \overline{D(A)}\right)$ and each bounded set $\mathcal{V}$ in $C_{\mathrm{b}}\left(\mathbb{R}_{+} ; \overline{D(B)}\right)$ which is relatively compact in $\widetilde{C}_{\mathrm{b}}([a,+\infty) ; Y)$, the set $q(\mathcal{U}, \mathcal{V})$ is relatively compact in $C\left(\left[-\tau_{2}, 0\right] ; Y\right)$.

Theorem 3.1. Let $\tau_{i} \geq 0, i=1,2$, and let $X$ and $Y$ be real Banach spaces. If $\left(\mathrm{H}_{A}\right),\left(\mathrm{H}_{B}\right),\left(\mathrm{H}_{F}\right),\left(\mathrm{H}_{G}\right),\left(\mathrm{H}_{c}\right),\left(\mathrm{H}_{p}\right)$, and $\left(\mathrm{H}_{q}\right)$ are satisfied, then (1.1) has at least one $C^{0}$-solution

$$
(u, v) \in C_{\mathrm{b}}\left(\left[-\tau_{1},+\infty\right) ; \overline{D(A)}\right) \times C_{\mathrm{b}}\left(\left[-\tau_{2},+\infty\right) ; \overline{D(B)}\right),
$$

satisfying

$$
\left\{\begin{array}{l}
\|u\|_{C_{\mathrm{b}}\left(\left[-\tau_{1},+\infty\right) ; X\right)} \leq \frac{m}{\omega-\ell}+\left[\frac{\omega}{\omega-\ell}\left(\frac{1}{e^{\omega a}-1}+\frac{\ell}{\omega}\right)+1\right] \cdot m \\
\|v\|_{C_{\mathrm{b}}\left(\left[-\tau_{2},+\infty\right) ; Y\right)} \leq \frac{m}{\gamma-\ell}+\left[\frac{\gamma}{\gamma-\ell}\left(\frac{1}{e^{\gamma a}-1}+\frac{\ell}{\gamma}\right)+1\right] \cdot m .
\end{array}\right.
$$

REMARK 3.2. We emphasize that the nonresonance condition $\ell<\delta$ ensures the existence of global $C^{0}$-solutions. In fact, one can imagine various types of growth conditions which in the case of local initial conditions, i.e., $p(u, v)(t):=$ $\psi_{1}(t)$ and $q(u, v)(t)=\psi_{2}(t)$ for $t \in[-\tau, 0]$, allow us to get global solutions from local ones via traditional Zorn's Lemma type arguments. We notice however that for nonlocal problems, as far as we can see, such kind of maximality arguments do not work.

REMARK 3.3. It is easy to see that hypotheses $\left(\mathrm{p}_{1}\right),\left(\mathrm{p}_{2}\right),\left(\mathrm{q}_{1}\right)$ and $\left(\mathrm{q}_{2}\right)$ ensure that the function $p$ depends only on the restrictions of $u$ and $v$ to $[a, d]$, while the function $q$ depends only on the restrictions of $u$ and $v$ to $[a,+\infty)$.

REMark 3.4. We listed below some important specific cases for the function $q$ for which hypothesis $\left(\mathrm{H}_{q}\right)$ is satisfied:

(a) $q(u, v)$ depends only on $v, q(u, v)=q_{0}(v)$, where

$$
q_{0}: C_{\mathrm{b}}\left(\left[-\tau_{2},+\infty\right) ; \overline{D(B)}\right) \rightarrow C\left(\left[-\tau_{2}, 0\right] ; \overline{D(B)}\right)
$$

is a continuous function such that $\left\|q_{0}(v)-q_{0}(\widetilde{v})\right\|_{C\left(\left[-\tau_{2}, 0\right] ; Y\right)} \leq\|v-\widetilde{v}\|_{C_{\mathrm{b}}([a,+\infty) ; Y)}$, where $a>0$ is given by $\left(\mathrm{H}_{p}\right)$;

(b) $q(u, v)=q_{1}(C u, v)$, where $C: C_{\mathrm{b}}([a,+\infty) ; \overline{D(A)}) \rightarrow C_{\mathrm{b}}([a,+\infty) ; \overline{D(A)})$ is a compact and nonexpansive operator and the function

$$
q_{1}: C_{\mathrm{b}}\left(\left[-\tau_{1},+\infty\right) ; \overline{D(A)}\right) \times C_{\mathrm{b}}\left(\left[-\tau_{2},+\infty\right) ; \overline{D(B)}\right) \rightarrow C\left(\left[-\tau_{2}, 0\right] ; \overline{D(B)}\right),
$$


is continuous from its domain, endowed with the sup-norm topology on $C_{\mathrm{b}}\left(\left[-\tau_{1}\right.\right.$, $+\infty) ; X)$ and the locally convex topology on $\widetilde{C}_{\mathrm{b}}\left(\left[-\tau_{2},+\infty\right) ; Y\right)$, to $C\left(\left[-\tau_{2}, 0\right] ; Y\right)$ and there exist $\ell_{i}>0, i=1,2$, with $\ell_{1}+\ell_{2} \leq 1$ such that

$\left\|q_{1}(u, v)-q_{1}(\widetilde{u}, \widetilde{v})\right\|_{C\left(\left[-\tau_{2}, 0\right] ; Y\right)} \leq \ell_{1}\|u-\widetilde{u}\|_{C_{\mathrm{b}}([a,+\infty) ; X)}+\ell_{2}\|v-\widetilde{v}\|_{C_{\mathrm{b}}([a,+\infty) ; Y)}$

for each $(u, v),(\widetilde{u}, \widetilde{v}) \in C_{\mathrm{b}}\left(\left[-\tau_{1},+\infty\right) ; \overline{D(A)}\right) \times C_{\mathrm{b}}\left(\left[-\tau_{2},+\infty\right) ; \overline{D(B)}\right)$, where $a>0$ is given by $\left(\mathrm{H}_{p}\right)$.

REMARK 3.5. From Theorem 3.1, we can obtain as simple consequences existence results referring to various systems whose forcing terms are either of the form $F\left(t, u_{t}, v(t)\right)$ and $G\left(t, u_{t}, v(t)\right)$ or of the form $F\left(t, u(t), v_{t}\right)$ and $G\left(t, u(t), v_{t}\right)$ or even of the form $F(t, u(t), v(t))$ and $G(t, u(t), v(t))$ and, in each of the cases considered, $p$ and $q$ are accordingly defined.

\section{An auxiliary result}

Lemma 4.1. Let $A: D(A) \subseteq X \rightsquigarrow X$ be $m$-dissipative with $0 \in D(A), 0 \in A 0$ and let us assume that there exists $\omega>0$ such that $A+\omega I$ is dissipative. Let $\tau \geq 0$ and $g: C_{\mathrm{b}}([-\tau,+\infty) ; \overline{D(A)}) \rightarrow C([-\tau, 0] ; \overline{D(A)})$ be such that there exists $a>0$ such that

$$
\|g(u)-g(\widetilde{u})\|_{C([-\tau, 0] ; X)} \leq\|u-\widetilde{u}\|_{C_{\mathrm{b}}([a,+\infty) ; X)}
$$

for each $u, \widetilde{u} \in C_{\mathrm{b}}([-\tau,+\infty) ; \overline{D(A)})$. Then, for each $h \in L^{\infty}\left(\mathbb{R}_{+} ; X\right)$ the problem

$$
\begin{cases}u^{\prime}(t) \in A u(t)+h(t) & \text { for } t \in \mathbb{R}_{+}, \\ u(t)=g(u)(t) & \text { for } t \in[-\tau, 0],\end{cases}
$$

has a unique $C^{0}$-solution $u^{h} \in C_{\mathrm{b}}([-\tau,+\infty) ; \overline{D(A)})$ satisfying

$$
\left\|u^{h}\right\|_{C_{\mathrm{b}}([-\tau,+\infty) ; X)} \leq \frac{e^{\omega a}}{e^{\omega a}-1} m_{0}+\frac{2}{\omega}\|h\|_{L^{\infty}\left(\mathbb{R}_{+} ; X\right)},
$$

where $m_{0}=\|g(0)\|_{C([-\tau, 0] ; X)}$. Moreover, the mapping $h \mapsto u^{h}$ is Lipschitz from $L^{\infty}\left(\mathbb{R}_{+} ; X\right)$ to $C_{\mathrm{b}}([-\tau,+\infty) ; X)$ with Lipschitz constant $1 / \omega$, i.e.,

$$
\left\|u^{h}-u^{\widetilde{h}}\right\|_{C_{\mathrm{b}}([-\tau,+\infty) ; X)} \leq \frac{1}{\omega}\|h-\widetilde{h}\|_{L^{\infty}\left(\mathbb{R}_{+} ; X\right)}
$$

for each $h, \widetilde{h} \in L^{\infty}\left(\mathbb{R}_{+} ; X\right)$.

Proof. The existence and uniqueness part and the proof of (4.3) follow the same lines as in the proof of Lemma 6.1 in Vrabie [41].

So, we will focus our attention only on the proof of (4.4). Let $h$ and $\widetilde{h}$ be arbitrary in $L^{\infty}\left(\mathbb{R}_{+} ; X\right)$ and $u^{h}, u^{\widetilde{h}}$ be the corresponding $C^{0}$-solutions of (4.2). 
From (2.2), we deduce

$$
\begin{aligned}
\left\|u^{h}(t)-u^{\widetilde{h}}(t)\right\| & \leq e^{-\omega t}\left\|g\left(u^{h}\right)(0)-g\left(u^{\widetilde{h}}\right)(0)\right\|+\int_{0}^{t} e^{-\omega(t-s)}\|h(s)-\widetilde{h}(s)\| d s \\
& \leq e^{-\omega t}\left\|g\left(u^{h}\right)-g\left(u^{\widetilde{h}}\right)\right\|_{C([-\tau, 0] ; X)}+\frac{1-e^{-\omega t}}{\omega}\|h-\widetilde{h}\|_{L^{\infty}\left(\mathbb{R}_{+} ; X\right)}
\end{aligned}
$$

for each $t \in \mathbb{R}_{+}$. Using (4.1), it follows

(4.5) $\left\|u^{h}(t)-u^{\widetilde{h}}(t)\right\| \leq e^{-\omega t}\left\|u^{h}-u^{\widetilde{h}}\right\|_{C_{\mathrm{b}}([a,+\infty) ; X)}+\frac{1-e^{-\omega t}}{\omega}\|h-\widetilde{h}\|_{L^{\infty}\left(\mathbb{R}_{+} ; X\right)}$

for each $t \in \mathbb{R}_{+}$. From the nonlocal initial condition and (4.1), we have

$$
\left\|u^{h}-u^{\widetilde{h}}\right\|_{C([-\tau, 0] ; X)} \leq\left\|g\left(u^{h}\right)-g\left(u^{\widetilde{h}}\right)\right\|_{C([-\tau, 0] ; X)} \leq\left\|u^{h}-u^{\widetilde{h}}\right\|_{C_{\mathrm{b}}([a,+\infty) ; X)} .
$$

As far as $\left\|u^{h}-u^{\widetilde{h}}\right\|_{C_{\mathrm{b}}([-\tau,+\infty) ; X)}$ is concerned, there are only three possible cases.

Case 1. There exists $\bar{t} \in[-\tau, 0]$ such that

$$
\left\|u^{h}-u^{\widetilde{h}}\right\|_{C_{\mathrm{b}}([-\tau,+\infty) ; X)}=\left\|u^{h}(\bar{t})-u^{\widetilde{h}}(\bar{t})\right\| .
$$

From the above inequality it follows

$$
\left\|u^{h}-u^{\widetilde{h}}\right\|_{C_{\mathrm{b}}([-\tau,+\infty) ; X)}=\left\|u^{h}-u^{\widetilde{h}}\right\|_{C([-\tau, 0] ; X)}=\left\|u^{h}-u^{\widetilde{h}}\right\|_{C_{\mathrm{b}}([a,+\infty) ; X)}
$$

and so this case reduces to one of the following two.

Case 2. There exists $\bar{t} \in(0,+\infty)$ satisfying (4.6). Using (4.5) with $t$ replaced by $\bar{t}$, we get

$\left\|u^{h}-u^{\widetilde{h}}\right\|_{C_{\mathrm{b}}([-\tau,+\infty) ; X)} \leq e^{-\omega \bar{t}}\left\|u^{h}-u^{\widetilde{h}}\right\|_{C_{\mathrm{b}}([-\tau,+\infty) ; X)}+\frac{1-e^{-\omega \bar{t}}}{\omega}\|h-\widetilde{h}\|_{L^{\infty}\left(\mathbb{R}_{+} ; X\right)}$.

Hence

$$
\left(1-e^{-\omega \bar{t}}\right)\left\|u^{h}-u^{\widetilde{h}}\right\|_{C_{\mathrm{b}}([-\tau,+\infty) ; X)} \leq \frac{1-e^{-\omega \bar{t}}}{\omega}\|h-\widetilde{h}\|_{L^{\infty}\left(\mathbb{R}_{+} ; X\right)}
$$

and so (4.4) holds true.

Case 3. There is no point $\bar{t} \in[-\tau,+\infty)$ such that (4.6) holds true. Then, there exists $\left(t_{k}\right)_{k}$ in $(0,+\infty)$ with $\lim _{k} t_{k}=+\infty$ such that

$$
\lim _{k}\left\|u^{h}\left(t_{k}\right)-u^{\widetilde{h}}\left(t_{k}\right)\right\|=\left\|u^{h}-u^{\widetilde{h}}\right\|_{C_{\mathrm{b}}([-\tau,+\infty) ; X)} .
$$

Substituting $t$ by $t_{k}$ in (4.5), we deduce

$\left\|u^{h}\left(t_{k}\right)-u^{\widetilde{h}}\left(t_{k}\right)\right\| \leq e^{-\omega t_{k}}\left\|u^{h}-u^{\widetilde{h}}\right\|_{C_{\mathrm{b}}([-\tau,+\infty) ; X)}+\frac{1-e^{-\omega t_{k}}}{\omega}\|h-\widetilde{h}\|_{L^{\infty}\left(\mathbb{R}_{+} ; X\right)}$, and passing to the limit for $k \rightarrow+\infty$, we get (4.4). 
Now, let us consider the system

$$
\begin{cases}u^{\prime}(t) \in A u(t)+\varphi(t) & \text { for } t \in \mathbb{R}_{+}, \\ v^{\prime}(t) \in B v(t)+\psi(t) & \text { for } t \in \mathbb{R}_{+}, \\ u(t)=p(u, v)(t) & \text { for } t \in\left[-\tau_{1}, 0\right], \\ v(t)=q(u, v)(t) & \text { for } t \in\left[-\tau_{2}, 0\right],\end{cases}
$$

where $(\varphi, \psi) \in L^{\infty}\left(\mathbb{R}_{+} ; X\right) \times L^{\infty}\left(\mathbb{R}_{+} ; Y\right)$.

LEMma 4.2. If $\left(\mathrm{H}_{A}\right),\left(\mathrm{H}_{B}\right),\left(\mathrm{p}_{1}\right),\left(\mathrm{p}_{2}\right)$ in $\left(\mathrm{H}_{p}\right)$ and $\left(\mathrm{q}_{1}\right),\left(\mathrm{q}_{2}\right)$ in $\left(\mathrm{H}_{q}\right)$ are satisfied, then for each $(\varphi, \psi) \in L^{\infty}\left(\mathbb{R}_{+} ; X\right) \times L^{\infty}\left(\mathbb{R}_{+} ; Y\right)$, (4.7) has a unique $C^{0}$-solution $\left(u^{\varphi}, v^{\psi}\right) \in C_{\mathrm{b}}\left(\left[-\tau_{1},+\infty\right) ; \overline{D(A)}\right) \times C_{\mathrm{b}}\left(\left[-\tau_{2},+\infty\right) ; \overline{D(B)}\right)$. In addition, the mapping $(\varphi, \psi) \mapsto\left(u^{\varphi}, v^{\psi}\right)$ is Lipschitz continuous from $L^{\infty}\left(\mathbb{R}_{+} ; X\right) \times$ $L^{\infty}\left(\mathbb{R}_{+} ; Y\right)$ to $C_{\mathrm{b}}\left(\left[-\tau_{1},+\infty\right) ; \overline{D(A)}\right) \times C_{\mathrm{b}}\left(\left[-\tau_{2},+\infty\right) ; \overline{D(B)}\right)$, with Lipschitz constant $L=\delta^{-1}$, where $\delta:=\min \{\omega, \gamma\}>0$, both domain and range being endowed with the max-norm of the corresponding factors.

Proof. We rewrite (4.7) as an evolution equation subject to nonlocal initial conditions in the product space $Z=X \times Y$ endowed with the max-norm, i.e., $\|(u, v)\|:=\max \{\|u\|,\|v\|\}$. Namely, let us define $\mathcal{A}: D(\mathcal{A}) \subseteq Z \rightsquigarrow Z$ by

$$
\left\{\begin{array}{l}
D(\mathcal{A})=D(A) \times D(B), \\
\mathcal{A}(u, v)=(A u, B v), \quad(u, v) \in D(\mathcal{A}),
\end{array}\right.
$$

Since $A$ and $B$ are $m$-dissipative it follows that $\mathcal{A}$ is $m$-dissipative too. Moreover, $0 \in D(\mathcal{A}), 0 \in \mathcal{A} 0$, and $\mathcal{A}+\delta I$ is dissipative. Let $h: \mathbb{R}_{+} \rightarrow Z$ be defined by $h(t)=(\varphi(t), \psi(t))$ for each $t \in \mathbb{R}_{+}$.

Let us define the function $g: C_{\mathrm{b}}([0,+\infty) ; \overline{D(\mathcal{A})}) \rightarrow \overline{D(\mathcal{A})}$ by

$$
\left.g(u, v)=(p(u, v)(0), q(u, v)(0)) \quad \text { for each }(u, v) \in C_{\mathrm{b}}([0,+\infty) ; \overline{D(\mathcal{A}})\right) .
$$

Let $z=(u, v)$ and let us consider the problem with nonlocal initial condition

$$
\left\{\begin{array}{l}
z^{\prime}(t) \in \mathcal{A} z(t)+h(t), \quad t \in \mathbb{R}_{+}, \\
z(0)=g(z) .
\end{array}\right.
$$

Since $p$ and $q$ satisfy $\left(\mathrm{p}_{1}\right)$ and $\left(\mathrm{p}_{2}\right)$ in $\left(\mathrm{H}_{p}\right)$ and respectively $\left(\mathrm{q}_{1}\right)$ and $\left(\mathrm{q}_{2}\right)$ in $\left(\mathrm{H}_{q}\right)$, we deduce that the function $g$ satisfies hypothesis (4.1) in Lemma 4.1 with $\tau=0$. Accordingly, the non-delay problem (4.8) has a unique $C^{0}$-solution $z^{h}=$ $(\widetilde{u}, \widetilde{v}) \in C_{\mathrm{b}}([0,+\infty) ; \overline{D(\mathcal{A})})$ and the mapping $h \mapsto z^{h}$ is Lipschitz continuous from $L^{\infty}\left(\mathbb{R}_{+} ; Z\right)$ to the space $C_{\mathrm{b}}([0,+\infty) ; \overline{D(\mathcal{A})})$, with Lipschitz constant $\delta^{-1}$.

Let us define $(u, v) \in C_{\mathrm{b}}\left(\left[-\tau_{1},+\infty\right) ; \overline{D(A)}\right) \times C_{\mathrm{b}}\left(\left[-\tau_{2},+\infty\right) ; \overline{D(B)}\right)$ by

$$
u(t)= \begin{cases}p(\widetilde{u}, \widetilde{v})(t) & \text { for } t \in\left[-\tau_{1}, 0\right), \\ \widetilde{u}(t) & \text { for } t \in[0,+\infty),\end{cases}
$$




$$
v(t)= \begin{cases}q(\widetilde{u}, \widetilde{v})(t) & \text { for } t \in\left[-\tau_{2}, 0\right), \\ \widetilde{v}(t) & \text { for } t \in[0,+\infty) .\end{cases}
$$

From Remark 3.3, we deduce that $p(\widetilde{u}, \widetilde{v})=p(u, v)$ and $q(\widetilde{u}, \widetilde{v})=q(u, v)$. As a consequence, $(u, v)$ is the unique $C^{0}$-solution of (4.7). Since $h \mapsto z^{h}$ is Lipschitz continuous from $L^{\infty}\left(\mathbb{R}_{+} ; Z\right)$ to $C_{\mathrm{b}}([0,+\infty) ; \overline{D(\mathcal{A})})$, by $\left(\mathrm{p}_{2}\right)$ in $\left(\mathrm{H}_{p}\right)$ and $\left(\mathrm{q}_{2}\right)$ in $\left(\mathrm{H}_{q}\right)$, we easily deduce that the mapping $(\varphi, \psi) \mapsto(u, v)$ is Lipschitz continuous from $L^{\infty}\left(\mathbb{R}_{+} ; X\right) \times L^{\infty}\left(\mathbb{R}_{+} ; Y\right)$ to $C_{\mathrm{b}}\left(\left[-\tau_{1},+\infty\right) ; \overline{D(A)}\right) \times C_{\mathrm{b}}\left(\left[-\tau_{2},+\infty\right) ; \overline{D(B)}\right)$, as claimed.

We recall the next result which will be useful in the sequel.

Theorem 4.3. Let $A: D(A) \subseteq X \rightsquigarrow X$ be an m-dissipative operator, with $0 \in D(A), 0 \in A 0$, let $\omega>0$ such that $A+\omega I$ is dissipative and let $\tau \geq 0$. Let $\left\{f_{n}: \mathbb{R}_{+} \times C([-\tau, 0] ; \overline{D(A)}) \rightarrow X ; n \in \mathbb{N}\right\}$ be a family of continuous functions satisfying:

$\left(\mathrm{h}_{1}\right)$ there exists $\ell \in(0, \omega)$ such that $\left\|f_{n}(t, x)-f_{n}(t, y)\right\| \leq \ell\|x-y\|_{C([-\tau, 0] ; X)}$ for each $n \in \mathbb{N}$, each $t \in \mathbb{R}_{+}$and $x, y \in C([-\tau, 0] ; \overline{D(A)})$;

$\left(\mathrm{h}_{2}\right)$ there exists $m>0$ such that $\left\|f_{n}(t, 0)\right\| \leq m$ for each $n \in \mathbb{N}$ and each $t \in \mathbb{R}_{+}$

$\left(\mathrm{h}_{3}\right) \lim _{n} f_{n}(t, x)=f(t, x)$ uniformly for $t \in \mathbb{R}_{+}$(for $t$ in bounded intervals in $\left.\mathbb{R}_{+}\right)$and $x$ in bounded subsets in $C([-\tau, 0] ; \overline{D(A)})$.

Let $\left\{g_{n}: C_{\mathrm{b}}([-\tau,+\infty) ; \overline{D(A)}) \rightarrow C([-\tau, 0] ; \overline{D(A)}) ; n \in \mathbb{N}\right\}$ be a family of functions satisfying:

$\left(\mathrm{h}_{4}\right)$ there exists $a>0$ such that for each $n \in \mathbb{N}$ and $u, \widetilde{u} \in C_{\mathrm{b}}([-\tau,+\infty) ; \overline{D(A)})$ we have $\left\|g_{n}(u)-g_{n}(\widetilde{u})\right\|_{C([-\tau, 0] ; X)} \leq\|u-\widetilde{u}\|_{C_{\mathrm{b}}([a,+\infty) ; X)}$;

$\left(\mathrm{h}_{5}\right) \lim _{n} g_{n}(u)=g(u)$ uniformly for $u$ in bounded subsets in $C_{\mathrm{b}}([-\tau,+\infty) ; \overline{D(A)})$ (and $g$ is continuous from $\widetilde{C}_{\mathrm{b}}([-\tau,+\infty) ; \overline{D(A)})$ to $\left.C([-\tau, 0] ; \overline{D(A)})\right)$.

Let $\left(u_{n}\right)_{n}$ be the sequence of $C^{0}$-solutions of the problem

$$
\begin{cases}u_{n}^{\prime}(t) \in A u_{n}(t)+f_{n}\left(t, u_{n_{t}}\right) & \text { for } t \in \mathbb{R}_{+}, \\ u_{n}(t)=g_{n}\left(u_{n}\right)(t) & \text { for } t \in[-\tau, 0] .\end{cases}
$$

Then $\lim _{n} u_{n}=u$ in $C_{\mathrm{b}}([-\tau,+\infty) ; X) \quad\left(\right.$ in $\left.\widetilde{C}_{\mathrm{b}}([-\tau,+\infty) ; X)\right)$, where $u$ is the unique $C^{0}$-solution of the problem

$$
\begin{cases}u^{\prime}(t) \in A u(t)+f\left(t, u_{t}\right) & \text { for } t \in \mathbb{R}_{+}, \\ u(t)=g(u)(t) & \text { for } t \in[-\tau, 0] .\end{cases}
$$

The existence and uniqueness of $C^{0}$-solutions of (4.9) were established by Vrabie [41, Theorem 3.1] in the case in which the initial constraint functions 
have affine growth, i.e., there exists $m_{0}>0$ such that

$$
\left\|g_{n}(u)\right\|_{C([-\tau, 0] ; X)} \leq\|u\|_{C_{\mathbf{b}}\left(\mathbb{R}_{+} ; X\right)}+m_{0},
$$

for each $n \in \mathbb{N}$ and $u \in C_{\mathrm{b}}([-\tau,+\infty) ; \overline{D(A)})$, and by Burlică and Roşu [11, Theorem 3.1] in the case $m_{0}=0$. For the proof of the continuity result in the $C_{\mathrm{b}}([-\tau,+\infty) ; X)$ case with $m_{0}=0$ see Burlică, Roşu and Vrabie [13]. The proof in the $\widetilde{C}_{\mathrm{b}}([-\tau,+\infty) ; X)$ case and $m_{0}>0$ is very similar with that of the preceding one and therefore we do not give details.

\section{The proof of Theorem 3.1}

We will use a fixed point technique coupled with some compactness arguments. Let $(u, v)$ be arbitrary but fixed in $C_{\mathrm{b}}\left(\left[-\tau_{1},+\infty\right) ; \overline{D(A)}\right) \times C_{\mathrm{b}}\left(\left[-\tau_{2},+\infty\right)\right.$; $\overline{D(B)})$. For the beginning we will prove that the next auxiliary problem

$$
\begin{cases}\widetilde{u}^{\prime}(t) \in A \widetilde{u}(t)+F\left(t, \widetilde{u}_{t}, \widetilde{v}_{t}\right) & \text { for } t \in \mathbb{R}_{+}, \\ \widetilde{v}^{\prime}(t) \in B \widetilde{v}(t)+G\left(t, u_{t}, v_{t}\right) & \text { for } t \in \mathbb{R}_{+}, \\ \widetilde{u}(t)=p(\widetilde{u}, \widetilde{v})(t) & \text { for } t \in\left[-\tau_{1}, 0\right], \\ \widetilde{v}(t)=q(u, \widetilde{v})(t) & \text { for } t \in\left[-\tau_{2}, 0\right],\end{cases}
$$

has a unique $C^{0}$-solution $(\widetilde{u}, \widetilde{v}) \in C_{\mathrm{b}}\left(\left[-\tau_{1},+\infty\right) ; \overline{D(A)}\right) \times C_{\mathrm{b}}\left(\left[-\tau_{2},+\infty\right) ; \overline{D(B)}\right)$. To do this, we will use Lemma 4.1 and we deduce that the problem

$$
\begin{cases}\widetilde{v}^{\prime}(t) \in B \widetilde{v}(t)+G\left(t, u_{t}, v_{t}\right) & \text { for } t \in \mathbb{R}_{+}, \\ \widetilde{v}(t)=q(u, \widetilde{v})(t) & \text { for } t \in\left[-\tau_{2}, 0\right],\end{cases}
$$

has a unique $C^{0}$-solution $\widetilde{v} \in C_{\mathrm{b}}\left(\left[-\tau_{2},+\infty\right) ; \overline{D(B)}\right)$ satisfying

$$
\|\widetilde{v}\|_{C_{\mathrm{b}}\left(\left[-\tau_{2},+\infty\right) ; Y\right)} \leq \frac{e^{\gamma a}}{e^{\gamma a}-1} m+\frac{2}{\gamma}\left\|G\left(\cdot, u_{(\cdot)}, v_{(\cdot)}\right)\right\|_{C_{\mathrm{b}}\left(\mathbb{R}_{+} ; Y\right)} .
$$

Now, let us consider the problem

$$
\begin{cases}\widetilde{u}^{\prime}(t) \in A \widetilde{u}(t)+F\left(t, \widetilde{u}_{t}, \widetilde{v}_{t}\right) & \text { for } t \in \mathbb{R}_{+}, \\ \widetilde{u}(t)=p(\widetilde{u}, \widetilde{v})(t) & \text { for } t \in\left[-\tau_{1}, 0\right] .\end{cases}
$$

From Theorem 3.1, in Vrabie [41] we deduce that problem (5.3) has at least one $C^{0}$-solution $\widetilde{u} \in \widetilde{C}_{\mathrm{b}}\left(\left[-\tau_{1},+\infty\right) ; \overline{D(A)}\right)$ satisfying

$$
\|\widetilde{u}\|_{C_{\mathrm{b}}\left(\left[-\tau_{1},+\infty\right) ; X\right)} \leq \frac{m}{\omega-\ell}+\left[\frac{\omega}{\omega-\ell}\left(\frac{1}{e^{\omega a}-1}+\frac{\ell}{\omega}\right)+1\right] \cdot m .
$$

Hypotheses $\left(\mathrm{F}_{1}\right)$ in $\left(\mathrm{H}_{F}\right)$ and $\left(\mathrm{p}_{2}\right)$ in $\left(\mathrm{H}_{p}\right)$ prove the uniqueness of solution $\widetilde{u}$ of (5.3). Thus, problem (5.1) has a unique $C^{0}$-solution $(\widetilde{u}, \widetilde{v})$.

From (5.2), (5.4) and $\left(\mathrm{G}_{1}\right)$, it readily follows that for each pair $(u, v)$ belonging to the set $C_{\mathrm{b}}\left(\left[-\tau_{1},+\infty\right) ; \overline{D(A)}\right) \times C_{\mathrm{b}}\left(\left[-\tau_{2},+\infty\right) ; \overline{D(B)}\right)$ the pair $(\widetilde{u}, \widetilde{v})$, defined as above, belongs to $C_{\mathrm{b}}\left(\left[-\tau_{1},+\infty\right) ; \overline{D(A)}\right) \times C_{\mathrm{b}}\left(\left[-\tau_{2},+\infty\right) ; \overline{D(B)}\right)$. 
The considerations above show that we can define the operator $\Gamma$ from the locally convex space $\widetilde{C}_{\mathrm{b}}\left(\left[-\tau_{1},+\infty\right) ; \overline{D(A)}\right) \times \widetilde{C}_{\mathrm{b}}\left(\left[-\tau_{2},+\infty\right) ; \overline{D(B)}\right)$ into itself by

$$
\Gamma(u, v):=(\widetilde{u}, \widetilde{v})
$$

where $(\widetilde{u}, \widetilde{v})$ is the unique $C^{0}$-solution of (5.1). We will prove that $\Gamma$ satisfies the hypotheses of the Tychonoff Fixed Point Theorem 2.3 and thus it has at least one fixed point which is a $C^{0}$-solution of(5.1). More precisely we will prove that $\Gamma$ maps a suitably defined nonempty, closed, bounded and convex subset $\mathcal{C}$ in $\widetilde{C}_{\mathrm{b}}\left(\left[-\tau_{1},+\infty\right) ; \overline{D(A)}\right) \times \widetilde{C}_{\mathrm{b}}\left(\left[-\tau_{2},+\infty\right) ; \overline{D(B)}\right)$ into itself, is continuous and compact. We do this with the help of next lemma.

Lemma 5.1. Let us assume that $\left(\mathrm{H}_{A}\right),\left(\mathrm{H}_{B}\right),\left(\mathrm{H}_{F}\right),\left(\mathrm{H}_{G}\right),\left(\mathrm{H}_{c}\right),\left(\mathrm{H}_{p}\right)$, and $\left(\mathrm{H}_{q}\right)$ are satisfied and let us consider $\widetilde{m}>0$ such that

$$
\left\{\begin{array}{l}
\ell\left(\frac{e^{\gamma a}}{e^{\gamma a}-1} m+\frac{\widetilde{m}}{\gamma}\right)+m \leq \widetilde{m}, \\
r_{1} \leq r_{2}
\end{array}\right.
$$

where

$$
\left\{\begin{array}{l}
r_{1}:=\frac{m}{\omega-\ell}+\left[\frac{\omega}{\omega-\ell}\left(\frac{1}{e^{\omega a}-1}+\frac{\ell}{\omega}\right)+1\right] \cdot m, \\
r_{2}:=\frac{e^{\gamma a}}{e^{\gamma a}-1} m+\frac{\tilde{m}}{\gamma} .
\end{array}\right.
$$

Let us denote by $W:=\widetilde{C}_{\mathrm{b}}\left(\left[-\tau_{1},+\infty\right) ; \overline{D(A)}\right) \times \widetilde{C}_{\mathrm{b}}\left(\left[-\tau_{2},+\infty\right) ; \overline{D(B)}\right)$ and by

$$
\mathcal{C}:=\left\{(u, v) \in W ;\|u\|_{C_{\mathrm{b}}\left(\left[-\tau_{1},+\infty\right) ; X\right)} \leq r_{1},\|v\|_{C_{\mathrm{b}}\left(\left[-\tau_{2},+\infty\right) ; Y\right)} \leq r_{2}\right\} .
$$

Then $\mathcal{C}$ is nonempty, closed, and convex in $\widetilde{C}_{\mathrm{b}}\left(\left[-\tau_{1},+\infty\right) ; X\right) \times \widetilde{C}_{\mathrm{b}}\left(\left[-\tau_{2},+\infty\right) ; Y\right)$. In addition, the operator $\Gamma: W \rightarrow W$ defined by (5.5) maps $\mathcal{C}$ into itself, is continuous and $\Gamma(\mathcal{C})$ is relatively compact.

Proof. In view of $\left(\mathrm{H}_{c}\right)$, we can choose $\widetilde{m}>0$ such that we have (5.6). Obviously, $\mathcal{C}$ given by (5.8) is a nonempty, closed and convex set in $\widetilde{C}_{\mathrm{b}}\left(\left[-\tau_{1},+\infty\right) ; X\right) \times$ $\widetilde{C}_{\mathrm{b}}\left(\left[-\tau_{2},+\infty\right) ; Y\right)$. Let us prove that $\Gamma$ maps $\mathcal{C}$ into itself. To do this, let $(u, v) \in \mathcal{C}$ be arbitrary and $(\widetilde{u}, \widetilde{v})=\Gamma(u, v)$. So, $\widetilde{v}$ is the unique $C^{0}$-solution of the problem

$$
\begin{cases}\widetilde{v}^{\prime}(t) \in B \widetilde{v}(t)+G\left(t, u_{t}, v_{t}\right) & \text { for } t \in \mathbb{R}_{+}, \\ \widetilde{v}(t)=q(u, \widetilde{v})(t) & \text { for } t \in\left[-\tau_{2}, 0\right],\end{cases}
$$

and $\widetilde{u}$ is the unique $C^{0}$-solution of the problem

$$
\begin{cases}\widetilde{u}^{\prime}(t) \in A \widetilde{u}(t)+F\left(t, \widetilde{u}_{t}, \widetilde{v}_{t}\right) & \text { for } t \in \mathbb{R}_{+}, \\ \widetilde{u}(t)=p(\widetilde{u}, \widetilde{v})(t) & \text { for } t \in\left[-\tau_{1}, 0\right] .\end{cases}
$$


From (5.4), we have

$$
\|\widetilde{u}\|_{C_{\mathrm{b}}\left(\left[-\tau_{1},+\infty\right) ; X\right)} \leq \frac{m}{\omega-\ell}+\left[\frac{\omega}{\omega-\ell}\left(\frac{1}{e^{\omega a}-1}+\frac{\ell}{\omega}\right)+1\right] \cdot m:=r_{1} .
$$

From $\left(\mathrm{G}_{1}\right)$, we get

$$
\left\|G\left(t, u_{t}, v_{t}\right)\right\| \leq \ell \max \left\{r_{1}, r_{2}\right\}+m \leq \ell\left(\frac{e^{\gamma a}}{e^{\gamma a}-1} m+\frac{\widetilde{m}}{\gamma}\right)+m \leq \widetilde{m}
$$

for each $t \in \mathbb{R}_{+}$. Let us remark that, in view of (2.3) in Theorem 2.1 and (5.10), we obtain

$$
\begin{aligned}
\|\widetilde{v}(t)\| & \leq e^{-\gamma t}\|\widetilde{v}(0)\|+\int_{0}^{t} e^{-\gamma(t-s)}\left\|G\left(s, u_{s}, v_{s}\right)\right\| d s \\
& \leq e^{-\gamma t}\|q(u, \widetilde{v})\|_{C\left(\left[-\tau_{2}, 0\right] ; Y\right)}+\frac{\widetilde{m}}{\gamma}\left(1-e^{-\gamma t}\right)
\end{aligned}
$$

for each $t \in \mathbb{R}_{+}$. Using $\left(\mathrm{q}_{1}\right)$ and $\left(\mathrm{q}_{2}\right)$, we deduce that

$$
\|q(u, \widetilde{v})\|_{C\left(\left[-\tau_{2}, 0\right] ; Y\right)} \leq\|\widetilde{v}\|_{C_{\mathrm{b}}([a,+\infty) ; Y)}+m .
$$

So, for each $t \in \mathbb{R}_{+}$, we have

$$
\|\widetilde{v}(t)\| \leq e^{-\gamma t}\left(\|\widetilde{v}\|_{C_{\mathrm{b}}([a,+\infty) ; Y)}+m\right)+\frac{\widetilde{m}}{\gamma}\left(1-e^{-\gamma t}\right) .
$$

From this inequality we conclude that

$$
\|\widetilde{v}\|_{C_{\mathrm{b}}([a,+\infty) ; Y)} \leq \frac{m}{e^{\gamma a}-1}+\frac{\widetilde{m}}{\gamma} .
$$

Indeed, we distinguish between next two possible cases.

Case 1. There exists $\bar{t} \in[a,+\infty)$ such that

$$
\|\widetilde{v}(\bar{t})\|=\|\widetilde{v}\|_{C_{\mathrm{b}}([a,+\infty) ; Y)} .
$$

Setting $t=\bar{t}$ in (5.12) and recalling that $\bar{t} \geq a>0$, we deduce (5.13).

Case 2. If there is no $\bar{t} \in[a,+\infty)$ such that (5.14) holds true, then there exists $\left(t_{n}\right)_{n}$ in $[a,+\infty)$ with $\lim _{n} t_{n}=+\infty$ and

$$
\|\widetilde{v}\|_{C_{\mathrm{b}}([a,+\infty) ; Y)}=\lim _{n}\left\|\widetilde{v}\left(t_{n}\right)\right\| .
$$

Substituting $t$ by $t_{n}$ in (5.12) and letting $n \rightarrow+\infty$, we obtain again (5.13).

We prove next that

$$
\|\widetilde{v}\|_{C_{\mathrm{b}}\left(\left[-\tau_{2},+\infty\right) ; Y\right)} \leq r_{2},
$$

where $r_{2}$ is given by (5.7). If there exists $\bar{t} \in\left[-\tau_{2}, 0\right]$ such that $\|\widetilde{v}(\bar{t})\|=$ $\|\widetilde{v}\|_{C_{\mathrm{b}}\left(\left[-\tau_{2},+\infty\right) ; Y\right)}$, from (5.11) and (5.13), we get

$$
\|\widetilde{v}\|_{C_{\mathrm{b}}\left(\left[-\tau_{2},+\infty\right) ; Y\right)} \leq\|q(u, \widetilde{v})\|_{C\left(\left[-\tau_{2}, 0\right] ; Y\right)} \leq \frac{e^{\gamma a}}{e^{\gamma a}-1} m+\frac{\widetilde{m}}{\gamma}=r_{2} .
$$


If there exists $\bar{t}>0$ such that $\|\widetilde{v}(\bar{t})\|=\|\widetilde{v}\|_{C_{\mathrm{b}}\left(\left[-\tau_{2},+\infty\right) ; Y\right)}$, by (2.3) in Theorem 2.1 , we have

$$
\begin{aligned}
\|\widetilde{v}(\bar{t})\| & \leq e^{-\gamma \overline{\bar{t}}}\|\widetilde{v}(0)\|+\int_{0}^{\bar{t}} e^{-\gamma(\bar{t}-s)}\left\|G\left(s, u_{s}, v_{s}\right)\right\| d s \\
& \leq e^{-\gamma \bar{t}}\|\widetilde{v}\|_{C_{\mathbf{b}}\left(\left[-\tau_{2},+\infty\right) ; Y\right)}+\frac{\widetilde{m}}{\gamma}\left(1-e^{-\gamma \bar{t}}\right) .
\end{aligned}
$$

Since $\bar{t}>0$, we get $\|\widetilde{v}\|_{C_{\mathrm{b}}\left(\left[-\tau_{2},+\infty\right) ; Y\right)} \leq \widetilde{m} / \gamma \leq r_{2}$.

If for each $t \in\left[-\tau_{2},+\infty\right)$ we have $\|\widetilde{v}(t)\| \leq\|\widetilde{v}\|_{C_{\mathrm{b}}\left(\left[-\tau_{2},+\infty\right) ; Y\right)}$, then there exists $\left(t_{n}\right)_{n}$ in $(0,+\infty)$ with $\lim _{n} t_{n}=+\infty$ and $\|\widetilde{v}\|_{C_{\mathrm{b}}\left(\left[-\tau_{2},+\infty\right) ; Y\right)}=\lim _{n}\left\|\widetilde{v}\left(t_{n}\right)\right\|$. Setting $t=t_{n}$ in (5.12) and passing to the limit for $n \rightarrow+\infty$, we obtain again $\|\widetilde{v}\|_{C_{\mathrm{b}}\left(\left[-\tau_{2},+\infty\right) ; Y\right)} \leq \widetilde{m} / \gamma \leq r_{2}$. From (5.9) and (5.15), it follows that $\Gamma$ maps $\mathcal{C}$ into itself.

In the next step we prove that $\Gamma$ is continuous. Let $\left(\left(u_{n}, v_{n}\right)\right)_{n}$ be an arbitrary sequence in $\mathcal{C}$ and let $\left(\widetilde{u}_{n}, \widetilde{v}_{n}\right)=\Gamma\left(u_{n}, v_{n}\right)$ for each $n \in \mathbb{N}$. So, for each $n \in \mathbb{N}$, $\widetilde{v}_{n}$ is the unique $C^{0}$-solution of the problem

$$
\begin{cases}\widetilde{v}_{n}^{\prime}(t) \in B \widetilde{v}_{n}(t)+G\left(t, u_{n_{t}}, v_{n_{t}}\right) & \text { for } t \in \mathbb{R}_{+}, \\ \widetilde{v}_{n}(t)=q\left(u_{n}, \widetilde{v}_{n}\right)(t) & \text { for } t \in\left[-\tau_{2}, 0\right],\end{cases}
$$

while $\widetilde{u}_{n}$ is the unique $C^{0}$-solution of the problem

$$
\begin{cases}\widetilde{u}_{n}^{\prime}(t) \in A \widetilde{u}_{n}(t)+F\left(t, \widetilde{u}_{n_{t}}, \widetilde{v}_{n_{t}}\right) & \text { for } t \in \mathbb{R}_{+}, \\ \widetilde{u}_{n}(t)=p\left(\widetilde{u}_{n}, \widetilde{v}_{n}\right)(t) & \text { for } t \in\left[-\tau_{1}, 0\right] .\end{cases}
$$

We suppose that

$$
\begin{cases}\lim _{n} u_{n}=u & \text { in } \widetilde{C}_{\mathrm{b}}\left(\left[-\tau_{1},+\infty\right) ; X\right), \\ \lim _{n} v_{n}=v & \text { in } \widetilde{C}_{\mathrm{b}}\left(\left[-\tau_{2},+\infty\right) ; Y\right) .\end{cases}
$$

Then, for each $T>0$,

$$
\begin{cases}\lim _{n} u_{n_{t}}=u_{t} & \text { in } C\left(\left[-\tau_{1}, 0\right] ; X\right), \\ \lim _{n} v_{n_{t}}=v_{t} & \text { in } C\left(\left[-\tau_{2}, 0\right] ; Y\right),\end{cases}
$$

uniformly for $t \in[0, T]$. Taking into account that, by $\left(\mathrm{G}_{2}\right)$, the family of functions $\{G(t, \cdot, \cdot) ; t \in[0, T]\}$ is uniformly equicontinuous on $C\left(\left[-\tau_{1}, 0\right] ; \overline{D(A)}\right) \times$ $C\left(\left[-\tau_{2}, 0\right] ; \overline{D(B)}\right)$, we deduce that

$$
\lim _{n} G\left(t, u_{n_{t}}, v_{n_{t}}\right)=G\left(t, u_{t}, v_{t}\right) \quad \text { uniformly for } t \in[0, T] .
$$

As the sequence $\left(\left(u_{n}, v_{n}\right)\right)_{n}$ is bounded in the space $C_{\mathrm{b}}\left(\left[-\tau_{1},+\infty\right) ; X\right) \times$ $C_{\mathrm{b}}\left(\left[-\tau_{2},+\infty\right) ; Y\right)$ (see $\left.(5.18)\right)$, from $\left(\mathrm{G}_{1}\right)$ we conclude that the family $\{G(\cdot$, $\left.\left.u_{n_{(\cdot)}}, v_{n_{(\cdot)}}\right) ; n \in \mathbb{N}\right\}$ is uniformly bounded in $C_{\mathrm{b}}\left(\mathbb{R}_{+} ; Y\right)$, and so it is uniformly integrable in $L^{1}(0, k ; Y)$ for each $k=1,2, \ldots$ 
On the other hand, since $\left(u_{n}, \widetilde{v}_{n}\right) \in \mathcal{C}$ for each $n \in \mathbb{N}$, by $\left(\mathrm{q}_{1}\right)$, it follows that the set $\left\{q\left(u_{n}, \widetilde{v}_{n}\right) ; n \in \mathbb{N}\right\}$ is bounded in $C\left(\left[-\tau_{2}, 0\right] ; Y\right)$. Therefore $\left\{q\left(u_{n}, \widetilde{v}_{n}\right)(0) ; n \in \mathbb{N}\right\}$ is bounded in $Y$. Since the operator $B$ generates a compact semigroup, from Theorem 2.2 we deduce that the set of solutions $\left\{\widetilde{v}_{n} ; n \in \mathbb{N}\right\}$ of problem (5.16) is relatively compact in $C([\delta, k] ; Y)$ for each $k=1,2, \ldots$ and for each $\delta \in(0, k]$. So, $\left\{\widetilde{v}_{n} ; n \in \mathbb{N}\right\}$ is relatively compact in $\widetilde{C}_{\mathrm{b}}([\delta,+\infty) ; Y)$ for each $\delta>0$ and so in $\widetilde{C}_{\mathrm{b}}([a,+\infty) ; Y)$. Since, by $\left(\mathrm{q}_{3}\right)$, the set $\left\{q\left(u_{n}, \widetilde{v}_{n}\right) ; n \in \mathbb{N}\right\}$ is relatively compact in $C\left(\left[-\tau_{2}, 0\right] ; Y\right)$, we get that $\left\{q\left(u_{n}, \widetilde{v}_{n}\right)(0) ; n \in \mathbb{N}\right\}$ is relatively compact in $Y$. Now, from the second part in Theorem 2.2, we deduce that $\left\{\widetilde{v}_{n} ; n \in \mathbb{N}\right\}$ is relatively compact in $\widetilde{C}_{\mathrm{b}}\left(\mathbb{R}_{+} ; Y\right)$. Consequently, by $\left(q_{2}\right)$ in $\left(H_{q}\right)$, we easily conclude that it is relatively compact in $\widetilde{C}_{\mathrm{b}}\left(\left[-\tau_{2},+\infty\right) ; Y\right)$. Then it follows that there exists $\widetilde{v} \in C_{\mathrm{b}}\left(\left[-\tau_{2},+\infty\right) ; \overline{D(B)}\right)$ such that on a subsequence, at least, we have $\lim _{n} \widetilde{v}_{n}=\widetilde{v}$ in $\widetilde{C}_{\mathrm{b}}\left(\left[-\tau_{2},+\infty\right) ; Y\right)$. Furthermore, we can pass to the limit for $n \rightarrow+\infty$ in (5.16) and we deduce that $\widetilde{v}$ is the unique $C^{0}$-solution of the problem

$$
\begin{cases}\widetilde{v}^{\prime}(t) \in B \widetilde{v}(t)+G\left(t, u_{t}, v_{t}\right) & \text { for } t \in \mathbb{R}_{+}, \\ \widetilde{v}(t)=q(u, \widetilde{v})(t) & \text { for } t \in\left[-\tau_{2}, 0\right] .\end{cases}
$$

From Theorem 4.3 (the $\widetilde{C}_{\mathrm{b}}\left(\left[-\tau_{1},+\infty\right) ; X\right)$ case) with

$$
\left\{\begin{array}{l}
f_{n}(t, \cdot):=F\left(t, \cdot, \widetilde{v}_{n_{t}}\right) \\
g_{n}(\cdot)=p\left(\cdot, \widetilde{v}_{n}\right)
\end{array}\right.
$$

for each $n \in \mathbb{N}$, we deduce that there exists $\widetilde{u} \in \widetilde{C}_{\mathrm{b}}\left(\left[-\tau_{1},+\infty\right) ; \overline{D(A)}\right)$ such that the sequence of $C^{0}$-solutions of (5.17) satisfies $\lim _{n} \widetilde{u}_{n}=\widetilde{u}$ in $\widetilde{C}_{\mathrm{b}}\left(\left[-\tau_{1},+\infty\right) ; X\right)$, where $\widetilde{u}$ is the unique $C^{0}$-solution of the problem

$$
\begin{cases}\widetilde{u}^{\prime}(t) \in A \widetilde{u}(t)+F\left(t, \widetilde{u}_{t}, \widetilde{v}_{t}\right) & \text { for } t \in \mathbb{R}_{+}, \\ \widetilde{u}(t)=p(\widetilde{u}, \widetilde{v})(t) & \text { for } t \in\left[-\tau_{1}, 0\right] .\end{cases}
$$

Hence $\lim _{n}\left(\widetilde{u}_{n}, \widetilde{v}_{n}\right)=(\widetilde{u}, \widetilde{v})$ in $\widetilde{C}_{\mathrm{b}}\left(\left[-\tau_{1},+\infty\right) ; X\right) \times \widetilde{C}_{\mathrm{b}}\left(\left[-\tau_{2},+\infty\right) ; Y\right)$ and so $\Gamma$ is continuous.

In the next step, we will prove that $\Gamma(\mathcal{C})$ is relatively compact. Let $\left(\left(u_{n}, v_{n}\right)\right)_{n}$ be an arbitrary sequence in $\mathcal{C}$ and let $\left(\widetilde{u}_{n}, \widetilde{v}_{n}\right)=\Gamma\left(u_{n}, v_{n}\right)$ for each $n \in \mathbb{N}$. As before, we deduce that the set $\left\{\widetilde{v}_{n} ; n \in \mathbb{N}\right\}$ is relatively compact in $\widetilde{C}_{\mathrm{b}}([a,+\infty) ; Y)$. By $\left(\mathrm{q}_{3}\right)$ in $\left(\mathrm{H}_{q}\right),\left\{q\left(u_{n}, \widetilde{v}_{n}\right) ; n \in \mathbb{N}\right\}$ is relatively compact in $C\left(\left[-\tau_{2}, 0\right] ; Y\right)$, which means that there exists $w \in C\left(\left[-\tau_{2}, 0\right] ; \overline{D(B)}\right)$ such that $\lim _{n} q\left(u_{n}, \widetilde{v}_{n}\right)=w$ in $C\left(\left[-\tau_{2}, 0\right] ; Y\right)$. Clearly this shows that $\left\{q\left(u_{n}, \widetilde{v}_{n}\right)(0) ; n \in \mathbb{N}\right\}$ is relatively compact. Reasoning as before, by Theorem 1.7.7, $\left\{\widetilde{v}_{n} ; n \in \mathbb{N}\right\}$ is relatively compact in $\widetilde{C}_{\mathrm{b}}\left(\left[-\tau_{2},+\infty\right) ; Y\right)$. So, there exists $\widetilde{v} \in C_{\mathrm{b}}\left(\left[-\tau_{2},+\infty\right) ; \overline{D(B)}\right)$ such that on a subsequence, at least, we have $\lim _{n} \widetilde{v}_{n}=\widetilde{v}$ in $\widetilde{C}_{\mathrm{b}}\left(\left[-\tau_{2},+\infty\right) ; Y\right)$. By virtue 
of Theorem 4.3, it follows that there exists $\widetilde{u} \in \widetilde{C}_{\mathrm{b}}\left(\left[-\tau_{1},+\infty\right) ; \overline{D(A)}\right)$ such that $\lim _{n} \widetilde{u}_{n}=\widetilde{u}$ in $\widetilde{C}_{\mathrm{b}}\left(\left[-\tau_{1},+\infty\right) ; X\right)$ and this completes the proof.

We continue with the proof of Theorem 3.1.

Proof. By Lemma 5.1, it follows that $\mathcal{C}$ is a nonempty, convex and closed subset in a separated local convex space and $\Gamma: \mathcal{C} \rightarrow \mathcal{C}$ is a continuous operator, such that $\Gamma(\mathcal{C})$ is relatively compact. So, by the Tychonoff Fixed Point Theorem 2.3, $\Gamma$ has at least one fixed point $(u, v) \in \mathcal{C}$, which means that $\Gamma(u, v)=(u, v)$. Clearly, $(u, v)$ is a $C^{0}$-solution of the problem (1.1). Since $\|u\|_{C_{\mathrm{b}}\left(\left[-\tau_{1},+\infty\right) ; X\right)} \leq r_{1}$ in Lemma 5.1 , we deduce the first estimate in (3.1), while the second one follows the very same lines as those in Vrabie [41, Theorem 3.1].

\section{Application to a reaction-diffusion system in $L^{2}(\Omega)$}

Let $\Omega$ be a nonempty, bounded domain in $\mathbb{R}^{k}, k \geq 2$, with $C^{1}$ boundary $\Sigma$ and let $\tau_{i} \geq 0, i=1,2, \omega, \gamma>0$. We denote by $Q_{+}=\mathbb{R}_{+} \times \Omega, \Sigma_{+}=\mathbb{R}_{+} \times \Sigma$, $Q_{\tau_{i}}=\left[-\tau_{i}, 0\right] \times \Omega, i=1,2$. Let us consider two maximal-monotone operators $\alpha: D(\alpha) \subseteq \mathbb{R} \rightsquigarrow \mathbb{R}$ and $\beta: D(\beta) \subseteq \mathbb{R} \rightsquigarrow \mathbb{R}$ satisfying $0 \in \alpha(0), 0 \in \beta(0)$ and let $F, G: \mathbb{R}_{+} \times C\left(\left[-\tau_{1}, 0\right] ; L^{2}(\Omega)\right) \times C\left(\left[-\tau_{2}, 0\right] ; L^{2}(\Omega)\right) \rightarrow L^{2}(\Omega)$ be continuous. Let $d>b>\tau_{1}>0$, let $\mu_{i}, i=1,2$, be two positive $\sigma$-finite and complete measures on $\left[b-\tau_{i},+\infty\right)$ with $\operatorname{supp} \mu_{1} \subseteq\left[b-\tau_{1}, d\right]$ and let $k_{i}: \mathbb{R}_{+} \rightarrow \mathbb{R}_{+}$be in $L^{2}\left(\mathbb{R}_{+} ; \mu_{i}, \mathbb{R}_{+}\right)$with $\left\|k_{i}\right\|_{L^{2}\left(\mathbb{R}_{+} ; \mu_{i}, \mathbb{R}_{+}\right)} \leq 1, i=1,2$. Also, let $W_{i}: \mathbb{R} \rightarrow \mathbb{R}$ with $W_{i}(0)=0, i=1,2$, be two nonexpansive functions, let $\mathcal{C}: L^{2}(\Omega) \rightarrow \mathbb{R}$ be linear and continuous and let $\xi_{i}, i=1,2$, be bounded in $C\left(\left[-\tau_{i}, 0\right] ; L^{2}(\Omega)\right)$.

We consider the following reaction-diffusion system:

$$
\begin{cases}\frac{\partial u}{\partial t}(t, x)=\Delta u(t, x)-\omega u(t, x)+F\left(t, u_{t}, v_{t}\right)(x) & \text { in } Q_{+} \\ \frac{\partial v}{\partial t}(t, x)=\Delta v(t, x)-\gamma v(t, x)+G\left(t, u_{t}, v_{t}\right)(x) & \text { in } Q_{+} \\ -\frac{\partial u}{\partial \nu}(t, x) \in \alpha(u(t, x)), \quad-\frac{\partial v}{\partial \nu}(t, x) \in \beta(v(t, x)) & \text { on } \Sigma_{+} \\ u(t, x)=\int_{b}^{\infty} k_{1}(s) W_{1}(v(t+s, x)) u(t+s, x) d \mu_{1}(s)+\xi_{1}(t)(x) & \text { in } Q_{\tau_{1}} \\ v(t, x)=\int_{b}^{\infty} k_{2}(s) W_{2}(v(t+s, x)) \mathcal{C} u(t+s, \cdot) d \mu_{2}(s)+\xi_{2}(t)(x) & \text { in } Q_{\tau_{2}}\end{cases}
$$

where, as usual, $\Delta$ is the Laplace operator in the sense of distributions over $\Omega$ and $\partial u / \partial \nu$ is the outer normal derivative of $u$ on $\Sigma$. 
REMARK 6.1. The nonlocal conditions in (6.1) are very general and include some important specific cases. Namely, let $a:=b-\tau_{1}$ and let $\left(t_{n}\right)_{n}$ be an increasing sequence in $[b,+\infty)$, let $\left(a_{n}\right)_{n}$ be a sequence of positive numbers with

$$
\sum_{n=0}^{\infty} a_{n}^{2} \leq 1
$$

and let the kernel $k_{2}:[a,+\infty) \rightarrow \mathbb{R}_{+}$be defined by

$$
k_{2}(s):=a_{n} \text { for } s \in\left[t_{n}, t_{n+1}\right) \text { and } n=0,1, \ldots
$$

We define the operator $\mathcal{C}: L^{2}(\Omega) \rightarrow \mathbb{R}$ by

$$
\mathcal{C} z:=\frac{1}{|\Omega|} \int_{\Omega} z(y) d y
$$

for each $z \in L^{2}(\Omega)$, and the positive $\sigma$-finite and complete measure $\mu_{2}$ from $\mathcal{B}$, the class of Borel measurable subsets in $[a,+\infty)$ to $\mathbb{R}$, by

$$
\mu_{2}(E):=\operatorname{Card}\left(\left\{n \in \mathbb{N} ; t_{n} \in E\right\}\right) .
$$

Here, as usual, Card $(S)$ denotes the cardinal of the set $S$. Then the nonlocal condition for $v$

$$
v(t, x)=\frac{1}{|\Omega|} \sum_{n=0}^{\infty} a_{n} W_{2}\left(v\left(t+t_{n}, x\right)\right) \int_{\Omega} u\left(t+t_{n}, y\right) d y+\xi_{2}(t)(x), \quad(t, x) \in Q_{\tau_{2}},
$$

rewrites as

$$
v(t, x)=\int_{b}^{\infty} k_{2}(s) W_{2}(v(t+s, x)) \mathcal{C} u(t+s, \cdot) d \mu_{2}(s)+\xi_{2}(t)(x), \quad(t, x) \in Q_{\tau_{2}} .
$$

Likewise, in the case of the nonlocal condition for $u$, we may consider a similar nonlocal condition except that, in this case, one should assume that $\left(t_{n}\right)_{n}$ is an increasing sequence in $[a, d]$ with $d>a$.

The next theorem, which cannot be obtained from Theorem 3.1 in Burlică, Roşu and Vrabie [14] and which is the main result concerning system (6.1), is a consequence of Theorem 3.1.

ThEOREM 6.2. Let $\Omega$ be a nonempty, bounded, open subset in $\mathbb{R}^{k}, k \geq 2$, with $C^{1}$ boundary $\Sigma$, let $\tau_{i} \geq 0, i=1,2, \omega, \gamma>0, d>b>\tau_{1}>0$ and let $a=b-\tau_{1}$ be given constants. Let $\alpha: D(\alpha) \subseteq \mathbb{R} \rightsquigarrow \mathbb{R}$ and $\beta: D(\beta) \subseteq \mathbb{R} \rightsquigarrow \mathbb{R}$ be two maximal monotone operators with $0 \in D(\alpha) \cap D(\beta), 0 \in \alpha(0) \cap \beta(0)$. Let $F, G: \mathbb{R}_{+} \times C\left(\left[-\tau_{1}, 0\right] ; L^{2}(\Omega)\right) \times C\left(\left[-\tau_{2}, 0\right] ; L^{2}(\Omega)\right) \rightarrow L^{2}(\Omega)$ be continuous and let $\mathcal{C}: L^{2}(\Omega) \rightarrow \mathbb{R}$ be a linear continuous functional. Let $\mu_{i}, i=1,2$, be two positive $\sigma$-finite and complete measures defined on the class of Borel measurable sets in $[a,+\infty)$, let $k_{i} \in L^{2}\left(\mathbb{R}_{+} ; \mu_{i}, \mathbb{R}_{+}\right)$, let $W_{i}: \mathbb{R} \rightarrow \mathbb{R}$ with $W_{i}(0)=0$ and let $\xi_{i} \in C\left(\left[-\tau_{i}, 0\right] ; L^{2}(\Omega)\right), i=1,2$. Let us assume that

$\left(\mathrm{h}_{1}\right) \operatorname{supp} \mu_{1} \subseteq[a, d]$; 
$\left(\mathrm{h}_{2}\right)$ there exist $\ell>0$ and $m \geq 0$ such that

$$
\begin{aligned}
& \|F(t, 0,0)\|_{L^{2}(\Omega)} \leq m, \\
& \quad\|F(t, u, v)-F(t, \widetilde{u}, \widetilde{v})\|_{L^{2}(\Omega)} \\
& \quad \leq \ell \max \left\{\|u-\widetilde{u}\|_{C\left(\left[-\tau_{1}, 0\right] ; L^{2}(\Omega)\right)},\|v-\widetilde{v}\|_{C\left(\left[-\tau_{2}, 0\right] ; L^{2}(\Omega)\right)}\right\} \\
& \quad\|G(t, u, v)\|_{L^{2}(\Omega)} \leq \ell \max \left\{\|u\|_{C\left(\left[-\tau_{1}, 0\right] ; L^{2}(\Omega)\right)},\|v\|_{C\left(\left[-\tau_{2}, 0\right] ; L^{2}(\Omega)\right)}\right\}+m \\
& \quad \text { for each }(t, u, v),(t, \widetilde{u}, \widetilde{v}) \in \mathbb{R}_{+} \times C\left(\left[-\tau_{1}, 0\right] ; L^{2}(\Omega)\right) \times C\left(\left[-\tau_{2}, 0\right] ; L^{2}(\Omega)\right) \\
& \left(\mathrm{h}_{3}\right) \text { the family of functions }\left\{G(t, \cdot, \cdot) ; t \in \mathbb{R}_{+}\right\} \text {is uniformly equicontinu } \\
& \quad \text { on } C\left(\left[-\tau_{1}, 0\right] ; L^{2}(\Omega)\right) \times C\left(\left[-\tau_{2}, 0\right] ; L^{2}(\Omega)\right) ; \\
& \left(\mathrm{h}_{4}\right)\left\|k_{i}\right\|_{L^{2}\left(\mathbb{R}_{+} ; \mu_{i}, \mathbb{R}\right)} \leq 1, i=1,2 ; \\
& \left(\mathrm{h}_{5}\right)\left|W_{i}(v)-W_{i}(\widetilde{v})\right| \leq|v-\widetilde{v}| \text { for each } v, \widetilde{v} \in \mathbb{R}, i=1,2 ; \\
& \left(\mathrm{h}_{6}\right)\left\|\xi_{i}\right\|_{C\left(\left[-\tau_{i}, 0\right] ; L^{2}(\Omega)\right)} \leq m \text { for } i=1,2 ; \\
& \left(\mathrm{h}_{7}\right)\|\mathcal{C}\| \leq 1 .
\end{aligned}
$$$$
\text { for each }(t, u, v),(t, \widetilde{u}, \widetilde{v}) \in \mathbb{R}_{+} \times C\left(\left[-\tau_{1}, 0\right] ; L^{2}(\Omega)\right) \times C\left(\left[-\tau_{2}, 0\right] ; L^{2}(\Omega)\right) ;
$$

$\left(\mathrm{h}_{3}\right)$ the family of functions $\left\{G(t, \cdot, \cdot) ; t \in \mathbb{R}_{+}\right\}$is uniformly equicontinuous

Let us assume also that $\ell<\min \{\omega, \gamma\}$. Then, (6.1) has at least one $C^{0}$-solution.

Proof. We rewrite system (6.1) in the abstract form (1.1) in $X=Y=$ $L^{2}(\Omega)$ and then we apply Theorem 3.1. Let us define $A: D(A) \subseteq L^{2}(\Omega) \rightarrow L^{2}(\Omega)$ by

$$
\left\{\begin{array}{l}
D(A):=\left\{u \in H^{2}(\Omega) \cap H^{1}(\Omega) ;-\frac{\partial u}{\partial \nu} \in \alpha(u) \text { on } \Sigma_{+}\right\}, \\
A u:=\Delta u-\omega u \text { for } u \in D(A),
\end{array}\right.
$$

and $B: D(B) \subseteq L^{2}(\Omega) \rightarrow L^{2}(\Omega)$ by

$$
\left\{\begin{array}{l}
D(B):=\left\{v \in H^{2}(\Omega) \cap H^{1}(\Omega) ;-\frac{\partial v}{\partial \nu} \in \beta(v) \text { on } \Sigma_{+}\right\}, \\
B v:=\Delta v-\gamma v \text { for } v \in D(B) .
\end{array}\right.
$$

Since $0 \in \alpha(0)$ and $0 \in \beta(0)$, it follows that $C_{0}^{\infty}(\Omega) \subseteq D(A)$ and $C_{0}^{\infty}(\Omega) \subseteq$ $D(B)$. So, $\overline{D(A)}=\overline{D(B)}=L^{2}(\Omega)$. Next, from Vrabie [36, Theorem 2.8.2, p. 77] we deduce that $A, A+\omega I, B$ and $B+\gamma I$ are $m$-dissipative on $L^{2}(\Omega), 0 \in$ $D(A) \cap D(B), 0=A(0)=B(0)$ and $A$ and $B$ generate compact semigroups. So, hypotheses $\left(\mathrm{H}_{A}\right)$ and $\left(\mathrm{H}_{B}\right)$ in Theorem 3.1 are satisfied. Further, assumptions $\left(\mathrm{h}_{2}\right),\left(\mathrm{h}_{3}\right)$ and $\left(\mathrm{h}_{7}\right)$ ensure that $F$ and $G$ satisfy $\left(\mathrm{H}_{F}\right)$ and respectively $\left(\mathrm{H}_{G}\right)$.

We define the nonlocal initial constraint functions

$$
\begin{gathered}
p: C_{\mathrm{b}}\left(\left[-\tau_{1},+\infty\right) ; L^{2}(\Omega)\right) \times C_{\mathrm{b}}\left(\left[-\tau_{2},+\infty\right) ; L^{2}(\Omega)\right) \rightarrow C\left(\left[-\tau_{1}, 0\right] ; L^{2}(\Omega)\right), \\
p(u, v)(t)(x):=\int_{b}^{\infty} k_{1}(s) W_{1}(v(t+s, x)) u(t+s, x) d \mu(s)+\xi_{1}(t)(x)
\end{gathered}
$$

for each $(u, v) \in C_{\mathrm{b}}\left(\left[-\tau_{1},+\infty\right) ; L^{2}(\Omega)\right) \times C_{\mathrm{b}}\left(\left[-\tau_{2},+\infty\right) ; L^{2}(\Omega)\right)$, for each $t \in$ $\left[-\tau_{1},+\infty\right)$ and for almost every for $x \in \Omega$ and

$$
q: C_{\mathrm{b}}\left(\left[-\tau_{1},+\infty\right) ; L^{2}(\Omega)\right) \times C_{\mathrm{b}}\left(\left[-\tau_{2},+\infty\right) ; L^{2}(\Omega)\right) \rightarrow C\left(\left[-\tau_{2}, 0\right] ; L^{2}(\Omega)\right),
$$




$$
q(u, v)(t)(x):=\int_{b}^{\infty} k_{2}(s) W_{2}(v(t+s, x)) C u(t+s, \cdot) d \mu(s)+\xi_{2}(t)(x)
$$

for each $(u, v) \in C_{\mathrm{b}}\left(\left[-\tau_{1},+\infty\right) ; L^{2}(\Omega)\right) \times C_{\mathrm{b}}\left(\left[-\tau_{2},+\infty\right) ; L^{2}(\Omega)\right)$, for each $t \in$ $\left[-\tau_{2},+\infty\right)$ and for almost every $x \in \Omega$. From $\left(\mathrm{h}_{4}\right)-\left(\mathrm{h}_{7}\right)$, we deduce that $p$ and $q$ satisfy $\left(\mathrm{H}_{p}\right)$ and $\left(\mathrm{H}_{q}\right)$. So, Theorem 3.1 applies, wherefrom the conclusion follows.

\section{REFERENCES}

[1] S. Aizicovici And H. LeE, Nonlinear nonlocal Cauchy problems in Banach spaces, Appl. Math. Lett. 18 (2005), 401-407.

[2] S. Aizicovici And M. MCKiBBen, Existence results for a class of abstract nonlocal Cauchy problems, Nonlinear Anal. 39 (2000), 649-668.

[3] S. Aizicovici And V. Staicu, Multivalued evolution equations with nonlocal initial conditions in Banach spaces, NoDEA Nonlinear Differential Equations Appl. 14 (2007), 361376.

[4] P. BARAs, Compacité de l'opérateur definissant la solution d'une équation d'évolution non linéaire $(d u / d t)+A u \ni f$, C. R. Math. Acad. Sci. Paris, 286 (1978), 1113-1116.

[5] V. BARBu, Nonlinear Differential Equations of Monotone Type in Banach Spaces, Springer Monographs in Mathematics, Springer Verlag, 2010.

[6] P. Benilan, Équations d'évolution dans un espace de Banach quelconque et applications, Thèse, Orsay (1972).

[7] O. Bolojan-Nica, G. Infante And R. Precup, Existence results for systems with nonlinear coupled nonlocal initial conditions, arXiv: 1309.3708v1 [math.CA], 2013.

[8] M. Burliç̆, Viability for multi-valued semilinear reaction diffusion systems, Ann. Acad. Rom. Sci. Ser. Math. 2 (2010), no. 1, 3-24.

[9] M. Burlic $\breve{A}$ And D. RoşU, A Viability result for semilinear reaction diffusion systems, An. Ştiinţ. Univ. Al. I. Cuza Iaşi (N.S.) 54 (2008), 361-382.

[10] _ The initial value and the periodic problems for a class of reaction diffusion systems, Dyn. Contin. Discrete Impuls. Syst. Ser. A Math. Anal. 15 (2008), 427-444.

[11] _ A class of nonlinear delay evolution equations with nonlocal initial conditions, Proc. Amer. Math. Soc. 142 (2014), 2445-2458.

[12] _ A class of reaction-diffusion systems with nonlocal initial conditions, An. Ştiint. Al. I. Cuza, Iaşi, (N.S.) LXI (2015), 59-78.

[13] M. Burlică, D. Roşu And I.I. Vrabie, Continuity with respect to the data for a delay evolution equation with nonlocal initial conditions, Libertas Math. (N.S.) 32 (2012), 3748.

[14] _ Abstract reaction diffusion systems with nonlocal initial conditions, Nonlinear Anal. 94 (2014), 107-119.

[15] L. Byszewski, Theorems about the existence and uniqueness of solutions of semilinear evolution nonlocal Cauchy problems, J. Math. Anal. Appl. 162 (1991), 494-505.

[16] T. Cardinali, R. Precup and P. Rubbioni, A unified existence theory for evolution equations and systems under nonlocal conditions, J. Math. Anal. Appl. 432 (2) (2014), DOI: $10.1016 /$ j.jmaa.2015.07.019.

[17] M.G. Crandall and T.M. Liggett, Generation of semi-groups of nonlinear transformations in general Banach spaces, Amer. J. Math. 93 (1971), 265-298.

[18] K. Deng, Exponential decay of solutions of semilinear parabolic equations with initial boundary conditions, J. Math. Anal. Appl. 179 (1993), 630-637. 
[19] J.I. DÍAZ AND I.I. VRABIE, Existence for reaction diffusion systems: A compactness method approach, J. Math. Anal. Appl. 188 (1994), 521-540.

[20] R.E. EDWARDs, Functional analysis theory and applications, Holt, Rinehart and Winston, New York Chicago San Francisco Toronto London, 1965.

[21] J. GARCíA-FALSET, Existence results and asymptotic behaviour for nonlocal abstract Cauchy problems, J. Math. Anal. Appl. 338 (2008), 639-652.

[22] J. GARCíA-FALSET AND S. REICH, Integral solutions to a class of nonlocal evolution equations, Comm. Contemp. Math. 12 (2010), 1032-1054.

[23] J. HALE, Functional differential equations, Applied Mathematical Sciences 3, Springer Verlag, 1971.

[24] G. Infante And M. MaCiEJeWski, Multiple positive solutions of parabolic systems with nonlinear, nonlocal initial conditions, arXiv: 1407.7497v1 [math.AP], 2014.

[25] M. McKibben, Discovering Evolution Equations with Applications, Vol. I Deterministic Models, Chapman \& Hall/CRC Appl. Math. Nonlinear Sci. Ser. (2011)

[26] E. Mitidieri AND I.I. VRABIE, Existence for nonlinear functional differential equations, Hiroshima Math. J. 17 (1987), 627-649.

[27] _ A class of strongly nonlinear functionaldifferential equations, Ann. Mat. Pura Appl. (4) CLI (1988), 125-147.

[28] M. Necula And M. Popescu, A viability result for differential inclusions on graphs, An. Ştiinţ. Univ. Al. I. Cuza Iaşi (N.S.) LXI (2015), 41-58.

[29] M. Necula, M. Popescu And I.I. VRabie, Nonlinear delay evolution inclusions on graphs, Proceedings of the IFIP TC7/2013 on System Modeling and Optimization, Klagenfurt, Lecture Notes in Computer Science (B. Kaltenbacher, C. Heuberger, Ch. Pötze and F. Rendl, eds.) 2014, 207-216.

[30] M. Necula AND I.I. VRabie, A viability result for a class of fully nonlinear reaction diffusion systems, Nonlinear Anal. 69 (2008), 1732-1743.

[31] O. NicA, Nonlocal initial value problems for first order differential systems, Fixed Point Theory 13 (2012), no. 2, 603-612.

[32] A. PAICU AND I.I. VRABIE, A class of nonlinear evolution equations subjected to nonlocal initial conditions, Nonlinear Anal. 72 (2010), 4091-4100.

[33] D. RoşU, Viability for nonlinear multi-valued reaction-diffusion systems, NoDEA Nonlinear Differential Equations Appl. 17 (2010), 479-496.

[34] _ Viability for a nonlinear multi-valued system on locally closed graph, An. Ştiinț. Univ. Al. I. Cuza, Iaşi (N.S.) 56 (2010), 343-362.

[35] A. Tychonoff, Ein Fixpunktsatz, Math. Ann. 111 (1935), 767-776.

[36] I.I. VRABIE, Compactness methods for nonlinear evolutions, Second Edition, Pitman Monographs and Surveys in Pure and Applied Mathematics 75, Longman, 1995.

[37] _ Existence for nonlinear evolution inclusions with nonlocal retarded initial conditions, Nonlinear Anal. 74 (2011), 7047-7060.

[38] _ Existence in the large for nonlinear delay evolution inclusions with nonlocal initial conditions, J. Funct. Anal. 262 (2012), 1363-1391.

[39] _ Nonlinear retarded evolution equations with nonlocal initial conditions, Dynam. Systems Appl. 21 (2012), 417-440.

[40] _ Global solutions for nonlinear delay evolution inclusions with nonlocal initial conditions, Set-Valued Var. Anal., 20 (2012), 477-497.

[41] _ Delay evolution equations with mixed nonlocal plus local initial conditions, Commun. Contemp. Math. 17 (2015), DOI: 10.1142/S0219199713500351. 
[42] R.N. WANG AND P.X. ZHU, Non-autonomous evolution inclusions with nonlocal history conditions: Global integral solutions, Nonlinear Anal. 85 (2013), 180-191.

[43] L. ZhU AND G. LI, Nonlocal differential equations with multivalued perturbations in Banach spaces, Nonlinear Anal. 69 (2008), 2843-2850.

Manuscript received November 3, 2015 accepted May 12, 2015

Monica-Dana BurlicĂ

Department of Mathematics and Informatics

"G. Asachi" Technical University of Iaşi

Iaşi 700506, ROMANIA

E-mail address: monicaburlica@yahoo.com

Daniela Roşu

Department of Mathematics and Informatics

"G. Asachi" Technical University University of Iaşi

Iaşi 700506, ROMANIA

E-mail address: rosudaniela100@yahoo.com 